\title{
Surgical treatment of paraspinal schwannoma of left supraclavicular fossa: a case report
}

\author{
Hai ZHANG ${ }^{1 *+}\left(\mathbb{D}\right.$, Wanli $\mathrm{LIN}^{1+}$, Hui $\mathrm{LI}^{2}$, Fengyuan PENG ${ }^{1}$, Weibi $\mathrm{CHE}^{1}$
}

\begin{abstract}
The incidence of paraspinal schwannoma of supraclavicular fossa is low, and its operative plan and complications have not been systematic reported. Here, we reported a case of supraclavicular paraspinal schwannoma in our center. The operative methods, intraoperative precautions and postoperative complications were illustrated. A 54 years old male patient was admitted to hospital due to pain in the left chest and back for 20 days. MRI showed $32 \times 68 \times 38 \mathrm{~mm}$ tumor adjacent to the spine of the left supraclavicular fossa. The patient underwent supraclavicular incision surgery, and the postoperative pathology showed schwannoma. After the operation, the nerve of the left upper limb was damaged, which was manifested as numbness in the skin above the joint of the left thumb, left index finger, middle finger, ring finger and little finger. The skin above the second finger joint of the little finger is numb and the grip strength is weakened. One month after operation, the symptoms were relieved. Surgery is the first choice for the treatment of supraclavicular tumors. It is necessary to review the anatomy of supraclavicular region in detail before the operation, and to protect important nerves and vessels during the operation.
\end{abstract}

Keywords: schwannoma; supraclavicular fossa; paraspinal; case report.

Practical Application: Supraclavicular schwannoma is a rare disease. Surgical resection is still the main method for the treatment of this disease. Complete tumor resection and maximum protection of surrounding tissues are important for the success of the surgery. Therefore, the surgical approach should be planned according to the location and size of the tumor before surgery, and serious complications should be avoided during surgery, especially brachial plexus injury. Because the tumor's benign lesions, Surgeons should make appropriate choices for complete resection and avoiding serious complications.

\section{Introduction}

Supraclavicular schwannoma is a rare disease and its incidence accounts for less than $1 \%$ of the head and neck tumors. The patients usually had no clinical symptoms, while about $5 \%$ patients suffered from sensory or motor dysfunction of the ipsilateral upper limbs or chest and back pain due to nerve invasion (Safaee et al., 2015). Painless boundary clearance mass in supraclavicular region could be found by physical examination (Safaee et al., 2015). Cervical ultrasonography, CT and MRI were used to determine the character and origin of the tumor. It is suggested that fine needle aspiration biopsy should be performed to further differentiate benign tumor, primary malignant tumor or metastatic lymph node, but sometimes it could not completely distinguish the histological types (Delaroche et al., 2011; Keleş et al., 2018; Chen et al., 2019). Surgical resection is still the main method for the treatment of this disease. Supraclavicular approach is the often used in the surgery. Complete tumor resection and maximum protection of surrounding tissues are important for the success of the surgery. Although the incidence of supraclavicular neurilemmoma is low, it often causes surgeons to suffer from the special location and complexity of the disease. Here, we reported the operation and postoperative situation of a middle-aged male patient with schwannoma in the left supraclavicular fossa and discussed the selection of surgical approach and the prevention of complications.

\section{Case presentation}

A 54 years old male patient complained with left chest and back pain for 20 days was admitted to the Thoracic Surgery Department of our hospital on July 29, 2020. 20 days ago, the patient had pain in the left chest and back, obvious after deep breathing and activity. No distant radiation pain or obvious abnormality in left upper limb movement and sensation were found.

Physical examination showed that right hand blood pressure was 125/84 mmHg, left hand blood pressure was 129/84 mmHg. There was no limitation of neck activity, soft neck, no jugular vein distension and normal carotid pulse. The left supraclavicular fossa protruded about $5 \mathrm{~cm}$, the palpable boundary was clear, and the size was about $3 \times 3 \mathrm{~cm}$. There was no obvious swelling and skin ulceration on the surface. Laboratory examination of tumor markers by ELISA showed that CEA was $6.30 \mathrm{ng} / \mathrm{ml}$ (0-5.00 ng/ml) and SCC was $3.29 \mathrm{ng} / \mathrm{ml}(0-1.50 \mathrm{ng} / \mathrm{ml})$ (Figure 1). 
A

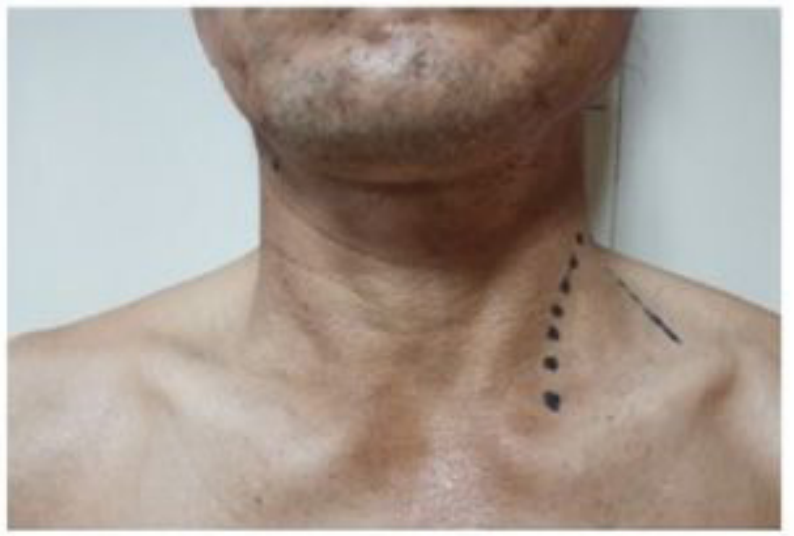

B

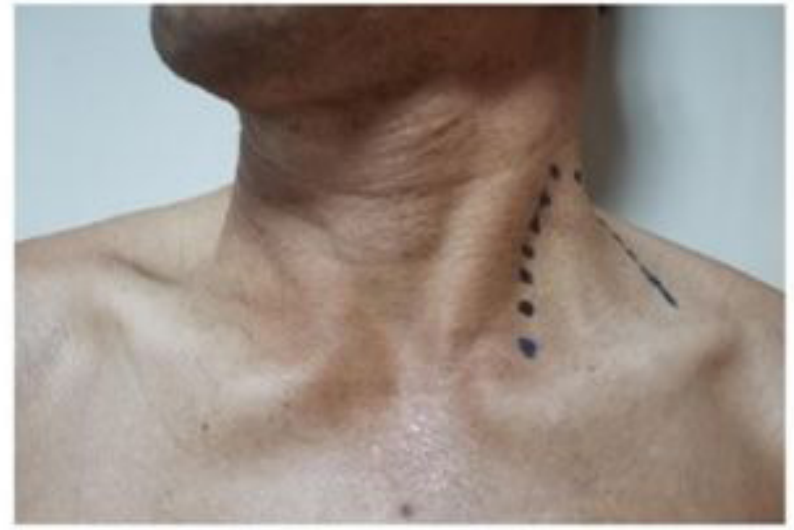

Figure 1. Physical examination. (A) Preoperative left supraclavicular mass (posteroanterior); (B) Preoperative left supraclavicular mass (lateral).

Chest CT plain scan + enhanced scan showed that an oval slightly low-density mass with a range of about $66 \mathrm{~mm} \times 30 \mathrm{~mm}$ was found at the left supraclavicular fossa paraspinal. The edge was slightly blurred and the density was uneven. The CT value was 15-39 HU. The boundary between the lesion and the left subclavian artery, and the left vertebral artery was not clear. The possibility of schwannoma should be considered for the left supraclavicular fossa paraspinal space occupying (Figure 2).

MRI plain scan + enhanced scan showed that on the left neck of C7-T2 plane, there was an irregular abnormal signal with clear boundary and uneven signal, showing long T1 and long T2 signal and high signal of lipid compression. On contrastenhanced scan, the lesion showed circular enhancement, which was connected with C7 / T1 intervertebral foramen nerve root, and the size of the lesion was about $32 \times 68 \times 38 \mathrm{~mm}$. The left cervical mass on C7-T2 plane is considered to be schwannoma (Figure 3). According to the CT imaging data of the patients, three-dimensional reconstruction was performed with mimics v21.0 (material's interactive medical image control system) (Figure 4). Based on the patient's history, physical examination and auxiliary examination, the patient was preliminary diagnosed with left supraclavicular fossa paraspinal space occupying, schwannoma? The patient underwent left supraclavicular paraspinal tumor resection via supraclavicular approach under general anesthesia with endotracheal intubation on August 6, 2020.

\section{Surgery}

The patient was placed in a supine position with $10 \mathrm{~cm}$ of shoulder height on the left side, and tracheal intubation was used for general anesthesia. A $12.0 \mathrm{~cm}$ incision was made along the upper edge of the left clavicle. The skin, subcutaneous tissue, platysma cervicis and anterior cervical fascia were incised successively. We cut the periosteum of the clavicle, cut off the clavicle at the middle point of the left clavicle, free it to both sides of the clavicle and pull it outward. The hyoid muscle of scapula was transected and the subclavian vein was dissociated. The space between the left subclavian vein and the internal jugular vein was separated until the tumor capsule. The brachial plexus was separated. The nerve retractor was pulled to avoid nerve injury. The upper and medial edges of the tumor were separated by blunt and sharp combination. The infraclavicular muscle was transected, and the space between the left subclavian vein and the upper edge of the first rib and the subclavian artery was separated until the capsule of the tumor. The lower and lateral edge of the tumor were separated by blunt and sharp method, and the tumor was removed from the anterior and posterior edges. Rapid frozen pathological examination showed that neurogenic tumor was considered. Blood leakage was found in the intervertebral foramen vein. Hemostatic gauze was used to stop bleeding for 15 minutes. The gauze was taken out till no further bleeding was found. The two broken ends of clavicle were fixed with clavicle embracing device. The drainage tube was placed about $3 \mathrm{~cm}$ under the left clavicle, and the vacuum bottle was connected outside. The incision was closed in turn. During the operation, a solid cystic mass was found near the spine of the left supraclavicular fossa, with the size of about $7 \mathrm{~cm}$ $\times 5 \mathrm{~cm} \times 5 \mathrm{~cm}$. The capsule was complete, soft and well-defined, containing about $20 \mathrm{ml}$ of light yellow liquid. The blood loss was $50 \mathrm{ml}$ and the vital signs were stable (Figure 5).

The drainage fluid of incision drainage tube was $30 \mathrm{ml}$, $30 \mathrm{ml}$ and $5 \mathrm{ml}$ respectively on the $1 \mathrm{st}, 2 \mathrm{nd}$ and $3 \mathrm{rd}$ day after operation. The drainage tube was removed on the 4 th day after operation. On the first day after operation, the patient developed skin numbness of the left thumb, left index finger, middle finger, ring finger and upside of the second knuckle of the little finger. The grip strength was weakened. On the 5 th day, electromyography of left upper limb showed that the amplitude of motor nerve conduction of left ulnar nerve was in normal range, and the latency was prolonged. The motor nerve conduction velocity, amplitude and latency of left median nerve were in normal range. The sensory nerve conduction velocity and amplitude of the left ulnar nerve decreased significantly. The sensory nerve conduction velocity of the left median nerve was in the normal range and the amplitude was decreased. 
A

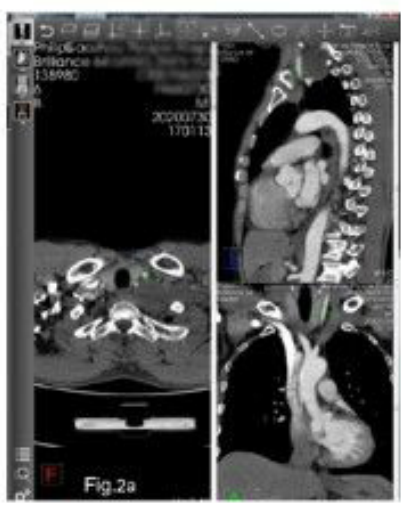

D

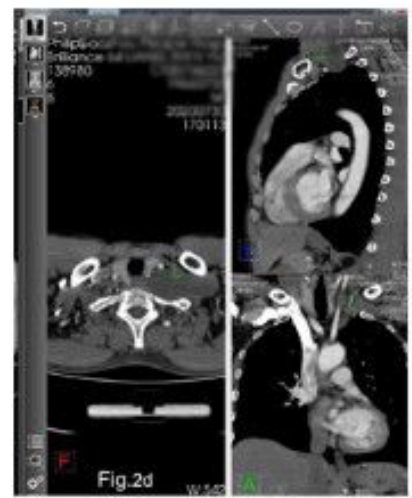

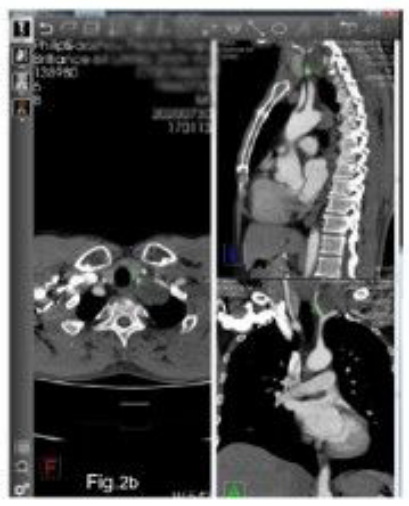

$\mathbf{E}$
C

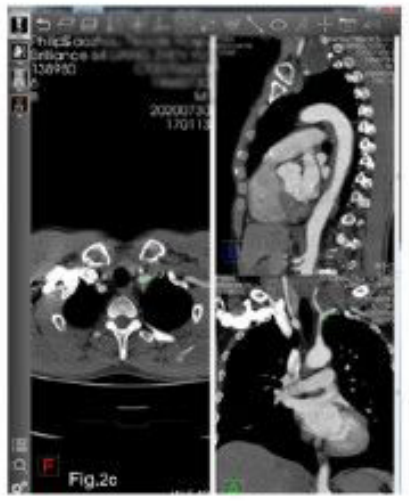

Figure 2. Chest CT plain scan + enhanced scan. (A) Left common carotid artery; (B) Left vertebral artery; (C) Subclavian artery; (D) Suprascapular artery; (E) Internal thoracic artery.

A

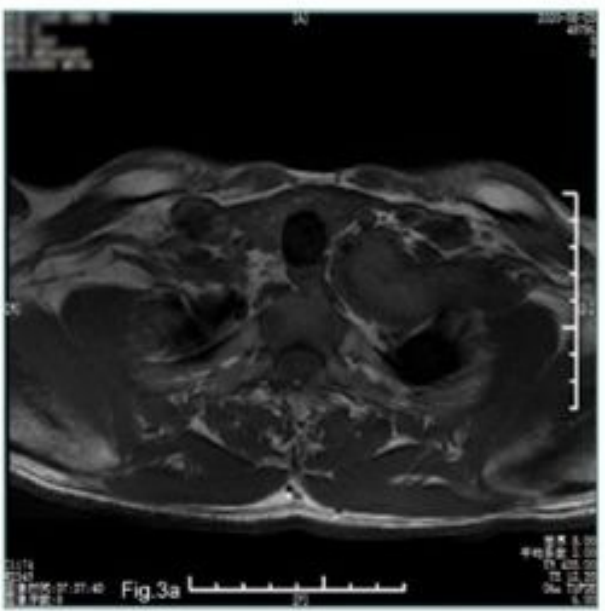

B

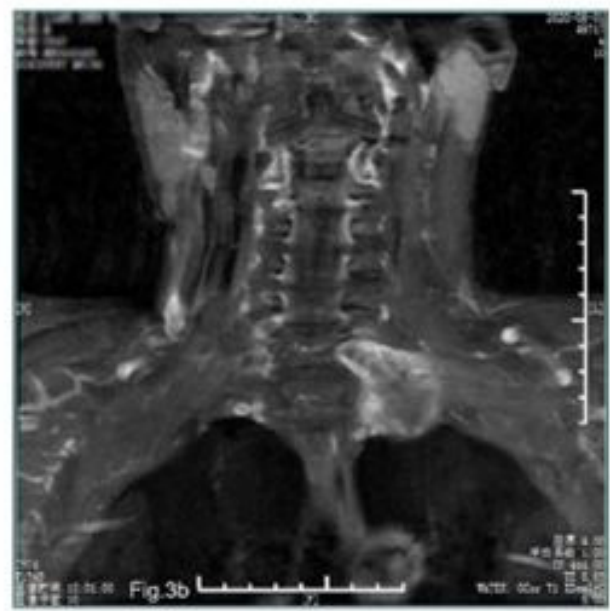

Figure 3. MRI plain scan + enhanced scan. (A) The lesion was connected with the nerve root of C7 / T1 intervertebral foramen (transection); (B) The lesion was connected with the nerve root of C7 / T1 intervertebral foramen (Longitudinal section). 


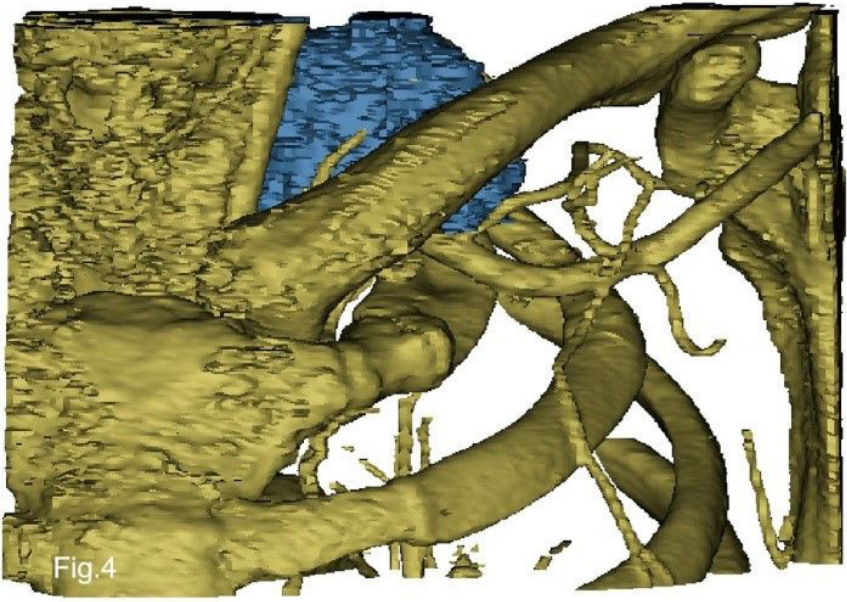

Figure 4. Three-dimensional reconstruction.
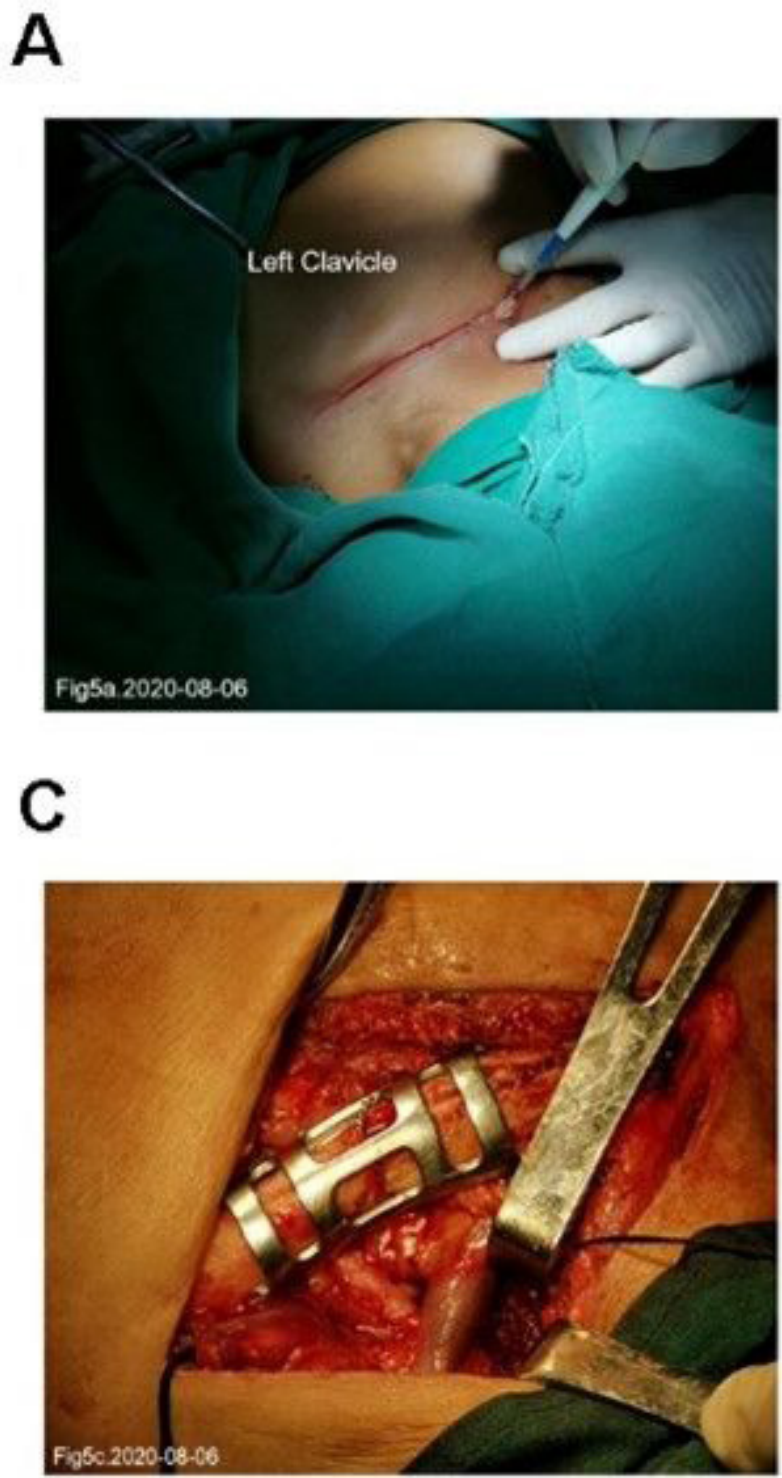

No spontaneous potential was found in the left abductor pollicis brevis and abductor pollicis pollicis, and the motor unit duration was not widened and the amplitude was increased. Therefore, the left upper limb was neurologically damaged. Postoperative pathological results showed cell rich schwannoma (Figure 6). The patient was discharged on the 9th day after operation. One month after the operation, the outpatient reexamination showed that the surgical incision healed in grade A (Figure 7). The movement of the left upper limb was not obviously limited. The numbness of the left upper limb and the symptoms of the left hand grip strength were improved. The patients will continue to follow up in the outpatient department of our center.

\section{Discussion}

Surgery is the first choice for the treatment of supraclavicular schwannoma. The goal of surgical treatment is complete resection

\section{B}

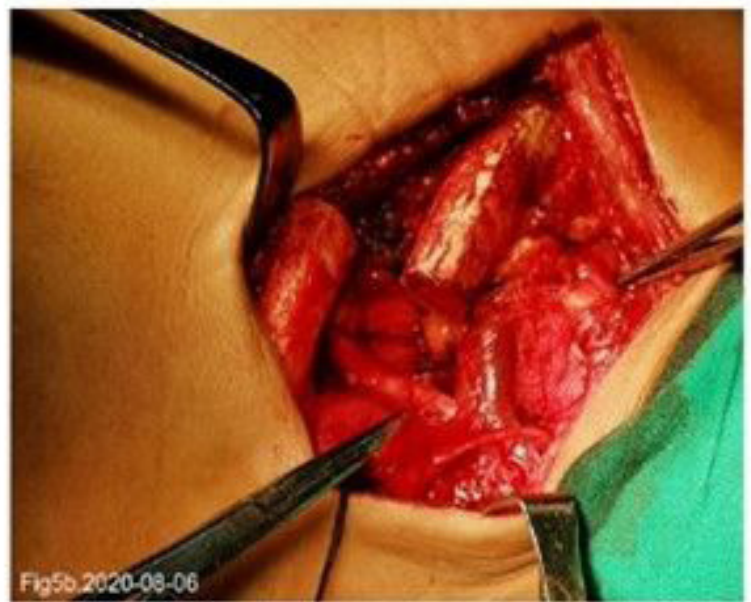

D

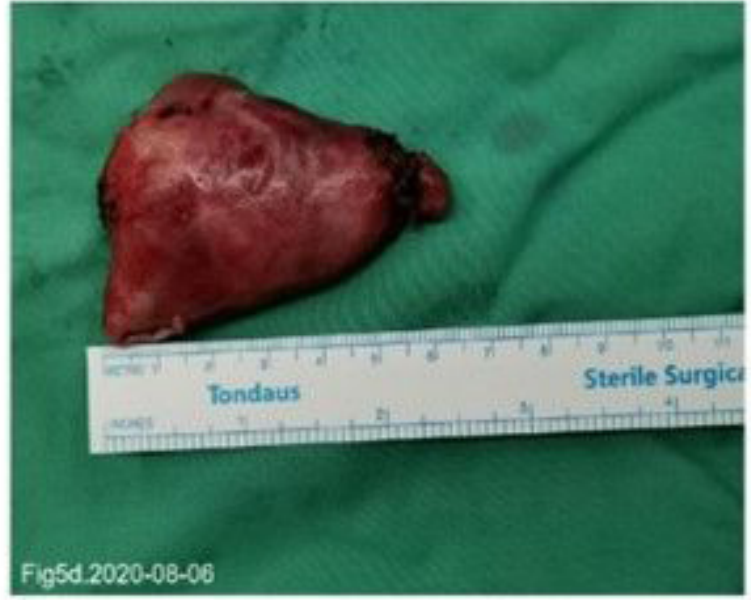

Figure 5. Surgery process. (A) Left supraclavicular incision approach; (B) Left subclavian artery, left internal jugular vein and brachial plexus nerve were exposed; (C) The anatomical structures after the left clavicle were reconstructed; (D) Gross observation of tumor. 
A

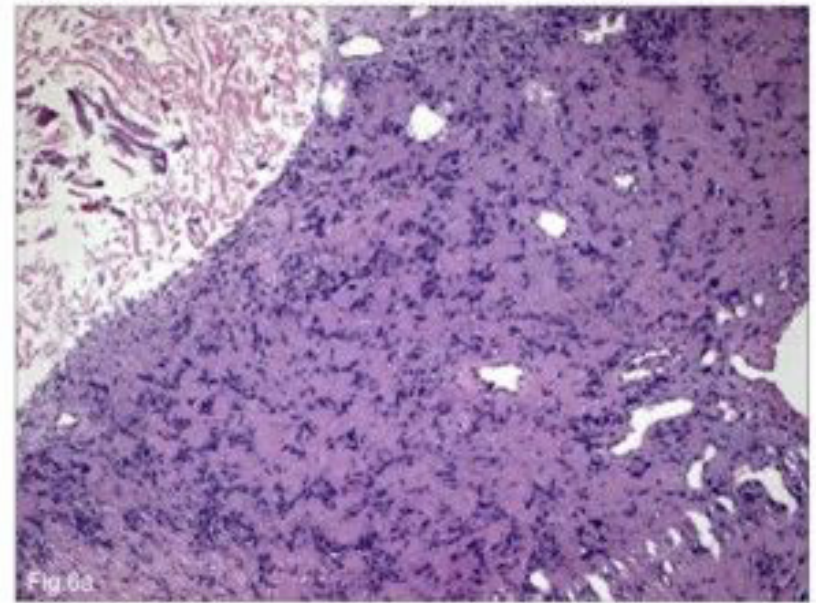

B

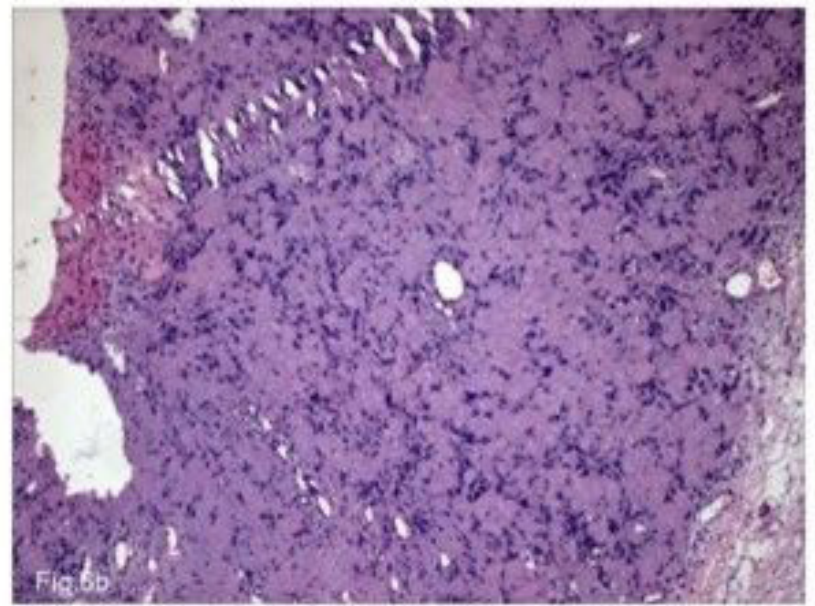

Figure 6. Pathological section of tumor. (A) The tumor was composed of Antinia A and B cells; (B) The cells were spindle or oval, arranged in palisade and chrysanthemum like clusters around the collagen fibers. The nuclei were stained deeply, the cytoplasm was few, and the mitotic figures were not found.

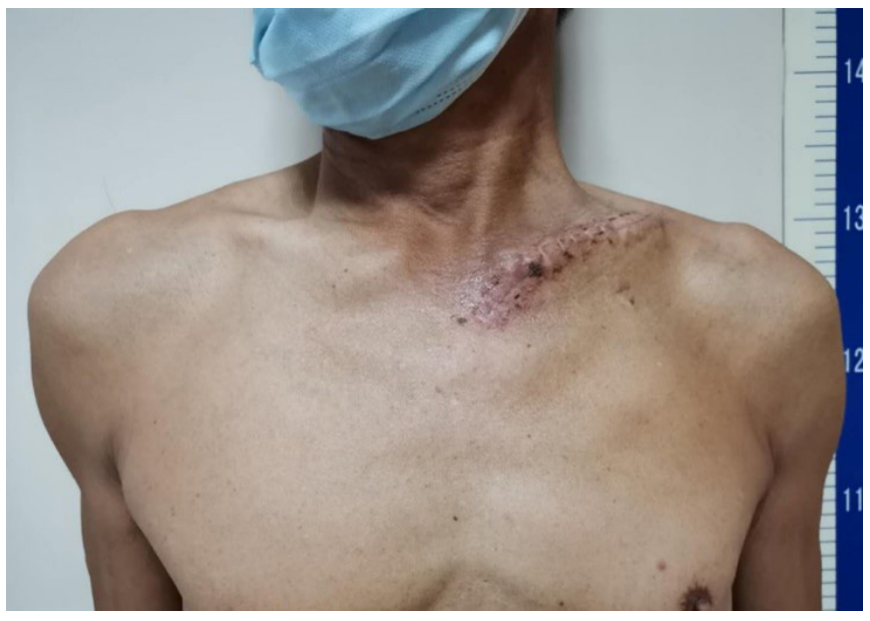

Figure 7. Follow-up one month after surgery.

and maximum protection of the surrounding tissue structure integrity. After operation, the recurrence rate of supraclavicular schwannoma was $7 \%$. The main reason for recurrence was the degree of complete resection due to the complexity of the anatomical site (Safaee et al., 2015; Fernandes et al., 2014; Conti et al., 2004). At present, most surgical incision was chosen in the supraclavicular plane in the surgical treatment of supraclavicular schwannoma and the integrity of the clavicle was retained. There is no report of cutting the clavicle in previous study. To fully expose the tumor and complete resection, we cut in the middle of clavicle in this patient. We consider that the indication for cutting the clavicle include, 1) The tumor was large, the length and diameter of the cross section was more than $6 \mathrm{~cm}$, and the solid component was the main component. If the tumor presents cystic structure, after the incision protection, sterilized injection needle can be used for needle aspiration or a small incision can be made in the safe area above the tumor body to absorb the internal liquid components, then the tumor body and the difficulty of operation can be reduced before complete tumor resection. 2) The boundary between the tumor and the left subclavian artery, and the initial segment of the left vertebral artery was not clear. 3 ) The right end of the tumor was located at C7-T2 plane and originated from C7 / T1 nerve root. More than $50 \%$ of the tumors were located below the midline of clavicle. The location was deep and the anatomical structure was complex. Supraclavicular incision alone could not reveal the tumor.

There were also some complications we needed to avoid in the surgery. First, phrenic nerve protection should be paid attention in this operation. The anterior part of phrenic nerve is adjacent to sternocleidomastoid muscle, scapulohyoid muscle intermediate tendon, internal jugular vein, transverse carotid artery and suprascapular artery, etc., and the left anterior is adjacent to thoracic duct arch. According to the anatomical characteristics of phrenic nerve, supraclavicular decompression is recommended for the treatment of neurogenic thoracic outlet syndrome. Meanwhile, if the phrenic nerve injury occurs, it should be repaired under the microscope (Sanders \& Annest, 2015). This patient had pain in the left chest and back before operation. Considering the compression of intercostal nerve caused by the large tumor, there was no obvious abnormality in the sensation and movement of the left upper limb, and there was no damage to the brachial plexus. Unfortunately, this patient developed numbness in his left upper limb on the first day after surgery, and EMG showed neurologic damage in his left upper limb. In previous study on surgical treatment of supraclavicular tumors, intraoperative electromyography could be used to identify nerves, thereby reducing or avoiding brachial plexus 
injury (Chen et al., 2019). Kindl et al. (2019) conducted detailed anatomy of brachial plexus during the operation of schwannoma originating from the supraclavicular fossa. If the tumor is excised directly on the medial side of sternocleidomastoid muscle without dissecting the brachial plexus one by one, though the chance of brachial plexus injury and operation time are reduced, the deep visual field cannot be better exposed due to the blind method, which may increase the risk of other unforeseen operations.

Second, for supraclavicular approach, attention should be paid to high chylous fistula or lymphatic fistula. The incidence of this risk is about $1-1.25 \%$, but more than $10 \%$ of the incidence has also been reported (Otero et al., 2010; Cheng \& Stoney, 1994). This patient's tumor was located under the jugular vein foot. When exposing the tumor, it is necessary to protect the thoracic duct and left bronchial mediastinal trunk, left jugular trunk and left subclavian trunk at the venous angle. For small lymphatic vessels, it is recommended to ligate them with No. 4 silk thread or use ultrasonic scalpel at low gear to prevent postoperative lymph leakage. At the same time, before closing the surgical incision, the surgical wound should be carefully examined. If the surgical wound is large, the incision drainage tube should be indwelled. In patients with postoperative drainage fluid and positive chylous test, they should take low-fat or even fat-free diet guidance and drug treatment according to the amount of drainage fluid. For refractory recurrent high-level chylous fistula, combined surgical treatment is needed (Tsang et al., 2020).

Third, the local anatomy of cervicothoracic junction is complex, and there are many large vessels and their branches. The risk of vascular injury is high. Any large vessel injury may lead to serious complications. For the large tumor, across the neck and chest, cervical thoracic incision or thoracoscope combined with neck incision could be chosen (Yamaguchi et al., 2006). In addition, it is suggested to perform the preoperative CT three-dimensional imaging on the site, or use the three-dimensional reconstruction software to reconstruct the site, so as to predict the high-risk area of surgical bleeding, and do careful operation to reduce the incidence of adverse events. For nerve root or intervertebral foramen hemorrhage, due to the deep location, narrow field of vision, unclear relationship of surrounding tissues, and close distribution of nerves, electrocoagulation is not recommended for hemostasis, but local compression hemostasis can be used. For patients with large amount of bleeding or difficult to stop bleeding, multi department combined operation was needed.

In conclusion, surgical resection of supraclavicular paraspinal tumors is feasible. The character of the tumor should be identified before operation, and the size, location and surrounding tissue structure of the tumor should be evaluated according to the imaging results to formulate an appropriate surgical approach. Surgeons should pay attention to the protection of nerve and blood vessels during the operation to avoid serious complications.

\section{Conflict of interest}

The author declare that they have no conflict of interest.

\section{References}

Chen, X., Ma, Q., Wang, S., Zhang, H., \& Huang, D. (2019). Surgical treatment of posterior mediastinal neurogenic tumors. Journal of Surgical Oncology, 119(6), 807-813. http://dx.doi.org/10.1002/ jso.25381. PMid:30653663.

Cheng, S. W., \& Stoney, R. J. (1994). Supraclavicular reoperation for neurogenic thoracic outlet syndrome. Journal of Vascular Surgery, 19(4), 565-572. http://dx.doi.org/10.1016/S0741-5214(94)70027-3. PMid:8164270.

Conti, P., Pansini, G., Mouchaty, H., Capuano, C., \& Conti, R. (2004). Spinal neurinomas: retrospective analysis and long-term outcome of 179 consecutively operated cases and review of the literature. Surgical Neurology, 61(1), 34-44. http://dx.doi.org/10.1016/S00903019(03)00537-8. PMid:14706374.

Delaroche, M., Gavilan-Cellié, I., Maurice-Tison, S., Kpozehouen, A., \& Dauman, R. (2011). Is behavioral audiometry achievable in infants younger than 6 months of age? International Journal of Pediatric Otorhinolaryngology, 75(12), 1502-1509. http://dx.doi.org/10.1016/j. ijporl.2011.08.016. PMid:21937124.

Fernandes, R. L., Lynch, J. C., Welling, L., Gonçalves, M., Tragante, R., Temponi, V., \& Pereira, C. (2014). Complete removal of the spinal nerve sheath tumors. Surgical technics and results from a series of 30 patients. Arquivos de Neuro-Psiquiatria, 72(4), 312-317. http:// dx.doi.org/10.1590/0004-282X20140008. PMid:24760097.

Keleş, E., Eroğlu, O., Özercan, İ. H., \& Özel, İ. (2018). Schwannoma in the supraclavicular region: case report. Turk Arch Otorhinolaryngol, 56(1), 54-57. http://dx.doi.org/10.5152/tao.2018.2579. PMid:29988291.

Kindl, R. P., Patel, K., \& Trivedi, R. A. (2019). Supraclavicular brachial plexus approach for excision of C8 nerve root Schwannoma: 3-dimensional operative video. Operative Neurosurgery, 16(5), 634-635. http://dx.doi.org/10.1093/ons/opy209. PMid:30101301.

Otero, M. J. L., López, M. T. F., Blanco, E. O., Vázquez, P. A., Osorio, I. P., \& Diz, D. I. (2010). Neck chylous fistula: conservative treatment. Nutrición Hospitalaria, 25(6), 1041-1044. PMid:21519779.

Safaee, M., Parsa, A. T., Barbaro, N. M., Chou, D., Mummaneni, P. V., Weinstein, P. R., Tihan, T., \& Ames, C. P. (2015). Association of tumor location, extent of resection, and neurofibromatosis status with clinical outcomes for 221 spinal nerve sheath tumors. Neurosurgical Focus, 39(2), E5. http://dx.doi.org/10.3171/2015.5.FOCUS15183. PMid:26235022.

Sanders, R. J., \& Annest, S. J. (2015). Technique of supraclavicular decompression for neurogenic thoracic outlet syndrome. Journal of Vascular Surgery, 61(3), 821-825. http://dx.doi.org/10.1016/j. jvs.2014.11.047. PMid:25600336.

Tsang, C. L. N., Gunanayagam, P., Feitosa, R., \& Villalba, L. (2020). High output chylous fistula post first rib resection. Annals of Vascular Surgery, 63, 455.e1-455.e5. http://dx.doi.org/10.1016/j. avsg.2019.07.023. PMid:31622760.

Yamaguchi, M., Yoshino, I., Kameyama, T., Osoegawa, A., Tagawa, T., \& Maehara, Y. (2006). Thoracoscopic surgery combined with a supraclavicular approach for removing a cervico-mediastinal neurogenic tumor: a case report. Annals of Thoracic and Cardiovascular Surgery, 12(3), 194-196. PMid:16823333. 